\title{
Neurology goes global Opportunities in international health
}

\author{
Jori E. Fleisher, MD \\ Farrah J. Mateen, MD, PhD
}

\begin{abstract}
Summary
In recent years, the need for additional neurologists and neurologic expertise in many low- and middleincome countries (LMIC) has become more apparent. Many organizations are committed to this unmet need, but the scope of the problem remains mostly underappreciated. Neurologists may be skeptical about their value in resource-limited settings, yet we are critically needed and can have a marked effect. International experiences, however, must be carried out in ethical, informed, and sustainable ways in tandem with local health care providers when possible. We present a brief overview of critical issues in global neurology, the importance of focusing on benefits to the LMIC, and options for volunteer opportunities in clinical service, education, research, and disaster relief. Finally, we offer practical pointers and resources for planning these experiences.
\end{abstract}

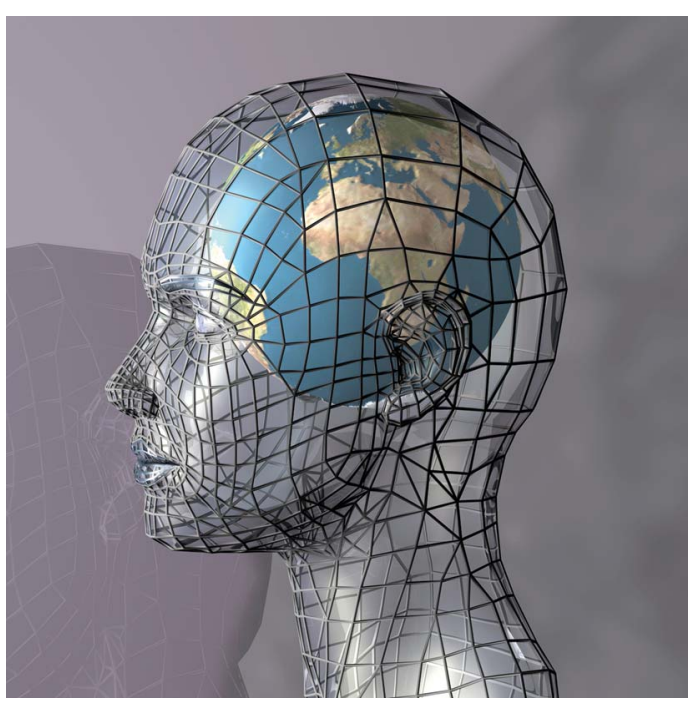

$\mathrm{T}$ he major health concerns in low- and middle-income countries (LMIC) have expanded beyond communicable, maternal, perinatal, and nutritional issues. The disability-adjusted life-years (DALYs) metric combines years of life lost due to premature mortality with years lived with disability to measure disease burden. ${ }^{1}$ Worldwide, noncommunicable diseases account for $54 \%$ of all DALYs, including hemorrhagic $(2.5 \%)$ and ischemic stroke (1.6\%), meningitis (1.2\%), migraine $(0.9 \%)$, epilepsy $(0.7 \%)$, and dementia $(0.5 \%)$. As illustrative examples, we briefly examine stroke and epilepsy.

While the incidence of stroke in high-income regions has declined by $42 \%$ over the past 4 decades, LMICs saw a $>100 \%$ increased incidence and 3.5-fold higher age-adjusted stroke mortality rates. The rising incidence follows the increased prevalence of stroke risk factors in LMICs, with hypertension being foremost. ${ }^{2}$ Limited stroke awareness and treatment availability prevent many patients from receiving acute stroke care. For survivors, secondary stroke prevention and rehabilitation facilities are severely limited or absent., ${ }^{2,3}$

Epilepsy is another common and undertreated condition globally. Up to $11 \%$ of people infected with HIV will develop epilepsy, ${ }^{4}$ most often secondary to opportunistic infections

Department of Neurology (JEF), University of Pennsylvania School of Medicine, Philadelphia; and the Department of Neurology (FJM), Massachusetts General Hospital, Boston.

Funding information and disclosures are provided at the end of the article. Full disclosure form information provided by the authors is available with the full text of this article at Neurology.org/cp.

Correspondence to: Jori.Fleisher@uphs.upenn.edu 
of the CNS, such as toxoplasmosis, tuberculosis (TB), and cryptococcal infection. ${ }^{5} \mathrm{~TB}$ meningitis carries an $8 \%-14 \%$ risk and cerebral malaria carries a $10 \%$ risk of developing epilepsy. ${ }^{5}$ Additionally, individuals in LMICs remain at risk for the inherited and acquired epilepsies seen in high-income countries. Despite the excess disease burden, over $80 \%$ of individuals with epilepsy in sub-Saharan African receive no treatment, due to scarce supplies, insufficient number of health care personnel, and stigma. ${ }^{5,6}$ Phenobarbital remains the most widely used antiepileptic drug in that region due to low cost and supply chain reliability; however, its efficacy must be balanced with the risk of drug interactions, particularly with antiretrovirals, antituberculous therapies, and hormonal contraceptives, as well as side effects and the occasional need for monitoring drug levels. $5,7,8$

In addition to the formidable disease burden, LMICs also face a severe shortage of local neurologists. A 2005 survey found 23 African nations averaging 1 neurologist per 5 million people, and 12 nations - nearly 26 million people - without any neurologists. ${ }^{9}$ Numerous efforts are underway to create or improve neurology training programs in LMICs. However, compounding the challenges in doing so is the "brain drain" of physicians leaving their home countries to practice in higher-income countries. ${ }^{3}$ This combination of high disease burden, limited diagnostic and treatment infrastructure, and a paucity of trained providers has produced a dramatic need for neurologic expertise in LMICs.

\section{Benefits for developing countries}

The ultimate goal of a neurology experience abroad should be improving the clinical care, clinical education, or research capacity for patients with neurologic diseases in the resource-limited setting. Capacity-building at a particular location requires partnerships between the visiting neurologist and local health care workers, sustained interactions before and after the in-person visit, and dedication to performing tasks that are solely for the benefit of the site. For neurologists who have trained in high-income settings, some tasks may be outside of one's traditional comfort zone since the subspecialized practice of neurology is uncommon elsewhere. Neurologic care in many countries is provided exclusively by nurses, psychiatrists, or traditional healers. Some ingrained practices may not be evidence-based and the balance struck between acceptance of unfamiliar protocols vs introduction of newer approaches must be carefully considered.

Some physicians and medical trainees have been accused of "medical tourism," which generally implies that the visiting physician has benefited more than the patients or health care workers on site. ${ }^{10}$ Although some effects of medical tourism may still be positive— such as improving intercultural awareness and introducing visiting physicians to low-income populations-the term is generally meant negatively. Well-intentioned but brief visits with inadequate understanding of local culture and limited involvement of local providers are often counterproductive; patients may be misunderstood and become distrustful of future visiting physicians.

LMICs may benefit from a neurologist's visit in several ways. Advocacy and attention to particular disorders, such as epilepsy or dementia, by a visiting neurologist may increase the local community's awareness of the disease. Visits may demonstrate to local leaders that neurologic disorders are common and require specialized care. They may also raise the profile of the work performed by local providers who have not received adequate recognition and support for their daily service to neurologic patients. Education of local providers, including allied health care workers and primary care providers, can improve patient care via a "train the trainer" approach. Provision of research support, including discussions of study design and medical literature, can be especially valuable to junior faculty in universities and health facilities abroad. Continuing 


\section{Sustained programming by an organization, even if individual physicians can only visit on a single occasion, is another viable approach to providing continuity of care and cooperation.}

medical education may also suit the needs of local physicians of various levels of experience and practice. Prioritizing locally relevant topics and providing English language support on completed manuscripts may be other ways to continue the relationship after the visit is complete.

\section{Opportunities in clinical service}

A recent review of US medical schools found that $24 \%$ offer a structured global health program, ${ }^{11}$ many of which are listed in table e-1 at Neurology.org/cp. For neurologists interested in a clinical experience, exploring the available programs at one's own academic institution or other nearby universities is a useful starting point. Although some programs limit participation to affiliated faculty only, many organizations will accept interested individuals following appropriate credentialing. Countless nongovernmental organizations (NGOs) and faith-based organizations exist to connect interested clinicians with medical volunteer opportunities; neurology-specific organizations are listed in table 1, with additional organizations offering general global health opportunities listed in table e-2.

Clinical service opportunities vary widely in duration, practice setting, and clinical responsibilities. Experiences can range from general practice to neurology-specific, from the treatment of helminthic infections and machete injuries to the provision of antiepileptics in the Ecuadorian rainforest. ${ }^{12}$ Short trips are the most susceptible to the drawbacks of medical tourism as above, but dedicated clinicians can minimize these risks by thorough pretrip preparation. Sustained programming by an organization even if individual physicians can only visit on a single occasion is another viable approach to providing continuity of care and cooperation.

Although fewer in number, longstanding collaborative partnerships exist, offering longer trips or a constant presence of rotating faculty members. Such alliances bear the greatest promise for sustainability by fostering teaching, research, and patient care. The Botswana-University of Pennsylvania Partnership, for example, offers residents the opportunity to spend 2 rotations at the same international site in the context of a longstanding collaboration between the United States and foreign institutions. Medical students, residents, and faculty are all eligible to spend time practicing within this context. In such a setting, less time and effort is necessary for each visiting clinician to build trust with local providers, so that truly collaborative patient care and teaching can start closer to the beginning of each trip. Longer trips can also provide opportunities to engage in outpatient or mobile clinics, distant outreach to rural villages, or home visits.

During 2 clinical experiences in Botswana, one author (J.F.) attended daily morning reports on the medical wards of the main referral hospital serving roughly 2 million people. Despite hosting the country's first and only medical school, there are no neurology faculty to care for the roughly $30 \%$ of medical ward admissions with neurologic chief complaints. Similar to the published experience of a colleague, ${ }^{13}$ roughly $30 \%$ of inpatient consults were for seizures, $20 \%$ each ischemic strokes and neuropathy, $10 \%$ intracranial hemorrhages, and $6 \%$ mass lesions. Historytaking required the cooperation of nursing staff and medical students as interpreters. CT scans were only intermittently available and films were frequently lost or unread for weeks. EEG, EMG, and other advanced neurodiagnostics were unavailable. Aspirin and generic antihypertensives and statins were on hand for stroke management. Periodically available antiepileptics included benzodiazepines, phenobarbital, phenytoin, valproic acid, and carbamazepine. In addition to inpatient consults, outpatients spanned the full range of neurology, from chronic migraineurs to individuals being seen for the first time for progressive supranuclear palsy, 
Table 1 Neurology-specific organizations with global interest

\begin{tabular}{|c|c|}
\hline Alliance for Stroke Awareness and Prevention Project & www.asapp.org \\
\hline Alzheimer's Disease International & www.alz.co.uk/1066 \\
\hline Brigada Neurologica to the Ecuadorian Amazon Rain Forest & www.cien-ecuador.org \\
\hline $\begin{array}{l}\text { CLIDEP: Clinique d'Epilepsie de Port-au-Prince (French or Creole } \\
\text { language skills required) }\end{array}$ & www.clidep.org \\
\hline European Federation of Neurological Societies & www.efns.org \\
\hline European Neurological Society & www.ensinfo.org \\
\hline Global Health Section, American Academy of Neurology & www.aan.com/go/about/sections/global \\
\hline Global NeuroCare & www.globalneurocare.org \\
\hline $\begin{array}{l}\text { Global neurology elective in Zambia; Beth Israel Deaconess Medical } \\
\text { Center }\end{array}$ & e-mail Dr. Omar Siddiqi: osiddiqi@bidmc.harvard.edu \\
\hline India Control Epilepsy & www.facebook.com/Epilepsylndia \\
\hline $\begin{array}{l}\text { Infectious and tropical neurology rotation in Kenya, Uganda, and } \\
\text { Morocco }\end{array}$ & e-mail Dr. David Renner: david.renner@hsc.utah.edu \\
\hline International Brain Research Organization & www.ibro.org \\
\hline International Bureau for Epilepsy & www.ibe-epilepsy.org \\
\hline International Child Neurology Association & www.icnapedia.org \\
\hline International League Against Epilepsy & www.ilae.org \\
\hline Lifting the Burden & www.l-t-b.org \\
\hline Movement Disorder Society Visiting Professor Program & www.movementdisorders.org/education/visiting/ \\
\hline Movement for Global Mental Health & www.globalmentalhealth.org \\
\hline $\begin{array}{l}\text { Neurology Capability Development, Oceania University of Medicine/ } \\
\text { Samoa National Health Service }\end{array}$ & $\begin{array}{l}\text { e-mail Drs. Viali Lameko or Logan McDaneld: } \\
\text { viali.lameko@oum.edu.ws; } \\
\text { Logan.mcdaneld@stmarygj.org }\end{array}$ \\
\hline Neurology residency rotation in Ethiopia & www.globalneurocare.org \\
\hline Neuropathic Pain Special Interest Group & www.neupsig.org \\
\hline Tropical neurology rotation in India & e-mail Dr. Mamta Singh: mbsneuro@gmail.com \\
\hline World Federation of Neurology & www.wfneurology.org \\
\hline World Neurology Foundation & www.worldneurology.org \\
\hline World Stroke Organization & www.world-stroke.org/ \\
\hline
\end{tabular}

amyotrophic lateral sclerosis, and Wilson disease. ${ }^{14}$ Without ancillary testing, the practice of neurology returns to the fundamentals of our field: a thorough history, careful examination, localization, diagnostic reasoning, and compassionate communication. One returns not only with honed clinical skills and gratitude for the patients and colleagues involved, but with a keener eye towards responsible, cost-effective care at home. ${ }^{15}$

\section{Opportunities in clinical education}

Clinical service trips frequently - and we would argue, should always-include associated clinical education, whether informal bedside teaching, grand rounds lectures, or clinical instruction to medical trainees. There are, however, many experiences focused primarily on education, such as the Visiting Professor Program offered by the Movement Disorders Society, or the Teaching Tools Workshops offered by the World Federation of Neurology (WFN). ${ }^{16}$ The WFN has an established protocol for providing educational programming to countries with unmet training needs. ${ }^{17}$ Based on a pilot program in Honduras, the WFN also created a set of detailed guidelines to be used in developing neurology residency programs abroad. ${ }^{17}$ 
Table 2 Pretravel resources

\begin{tabular}{|c|c|c|}
\hline Name & Web site & Contents \\
\hline CDC Traveler's Health & $\begin{array}{l}\text { www.cdc.gov/travel/page/yellowbook- } \\
\text { home-2012 }\end{array}$ & $\begin{array}{l}\text { Centers for Disease Control Guide to Traveler's Health, } \\
\text { including directory of vaccines and prophylaxis required } \\
\text { by country }\end{array}$ \\
\hline $\begin{array}{l}\text { Global Health } \\
\text { Education Consortium }\end{array}$ & Globalhealtheducation.org/resources & $\begin{array}{l}\text { Global health learning modules, preparing for overseas } \\
\text { experiences, handbooks for global engagement, global } \\
\text { health research, and career resources }\end{array}$ \\
\hline $\begin{array}{l}\text { "Neurology in Africa" } \\
\text { free online textbook }\end{array}$ & $\begin{array}{l}\text { www.uib.no/cih/en/resourcesneurology-in- } \\
\text { africa }\end{array}$ & $\begin{array}{l}\text { Free online practical neurology textbook written for } \\
\text { sub-Saharan Africa }\end{array}$ \\
\hline $\begin{array}{l}\text { US State Department } \\
\text { Travel Information }\end{array}$ & Travel.state.gov & $\begin{array}{l}\text { Tips for traveling abroad, updated travel alerts, information } \\
\text { on passports and visas, trip registration }\end{array}$ \\
\hline $\begin{array}{l}\text { WHO Global Health } \\
\text { Data \& Statistics }\end{array}$ & www.who.int/research/en/ & $\begin{array}{l}\text { Searchable directory of mortality and global health } \\
\text { estimates by region and nation }\end{array}$ \\
\hline
\end{tabular}

Due to the workforce issues in many LMICs, opportunities often involve teaching nonneurologist physicians or allied health professionals. Exceptional resources for such settings include "Where There Is No Neurologist" by Gretchen Birbeck, MD, MPH, and "Neurology in Africa" by William Howlett, MD. Both texts are freely available for download (table 2). When planning more formal lectures, practical issues are key: the availability and type of computers, projectors, electrical converters and adapters, and audience's level of medical training and English proficiency. As with all teaching, knowing one's audience is paramount, in terms of both the level of familiarity with the topic and the perceived need for such instruction. Finally, asking colleagues who have taught abroad whether they have already prepared lectures on the topics of interest and browsing the Global Health Education Consortium Web site for adaptable material are useful preparations. For the sake of time, collaboration, and optimal learning, we should avoid the tendency to recreate basic material and rather search for, improve upon, and share existing work.

\section{Opportunities in global neurology research}

Global research collaborations have made substantial contributions to the diagnosis and management of neurologic disease worldwide, and the funding for cooperative efforts has increased. ${ }^{18}$ Sources include the Fulbright and Fogarty Global Health Scholars Programs, American Academy of Neurology Research Training Fellowships, and Burroughs Wellcome Fund Fellowships in Tropical Diseases. The NIH supports Medical Education Partnership Initiatives, International Research Scientist Development Awards, and grants for Brain Disorders in the Developing World. Additional funding may be available from the academic institutions and organizations listed in tables e-1 and e-2. Neurologists interested in global neurology research are encouraged to establish strong collegial relationships with local providers, be mindful of sustainability, maintain the highest ethical standards, ${ }^{19}$ and acquaint themselves with local institutional review board policies in the earliest stages of planning.

\section{Opportunities in disaster relief}

The opportunity to provide disaster relief may not be anticipated and can include care following a variety of circumstances such as earthquakes, tornadoes, hurricanes, or armed violence. This may occur domestically, as in the recent cases of Hurricane Katrina or the Boston 


\section{Figure Pretravel checklist}

$>6$ Months before:

$\square$ Meet with institutional/departmental administration; secure time, coverage of responsibilities

$\square$ Explore funding opportunities

$\square$ Establish collegial relationship with host; set expectations

3-6 Months before:

$\square$ Determine entry and licensure requirements

$\square$ Obtain passport and visas, if necessary

$\square$ Book travel and housing

$\square$ Obtain necessary vaccinations or prophylaxis, medical insurance; establish medical evacuation plan

Find resources on local culture, language, medical system

Find local providers for referrals and patient follow-up

Read about basic neuroepidemiology of visit site

1 Month before:

$\square$ Confirm specifics of visit with home and visiting institutions

$\square$ Register travel with US State Department

$\square$ Review procedural, neuroradiology skills

$\square$ Practice neurological history \& examination in foreign language, if necessary

$\square$ Review diagnostic, therapeutic, ancillary services available at visit site in preparation

$\square$ Prepare lectures on selected applicable topics, if desired by host institution

End of/after visit:

$\square$ Confirm contact information for collaborators and colleagues

$\square$ Plan for debriefing with colleagues at home institution

bombing, or internationally in fragile states or locations in active armed conflict. Since more than 1.5 billion people live in postconflict regions, the chances of visiting a location with a history of armed conflict is high. In locations of protracted need, such as Haiti or refugee camps in the Middle East, NGOs and established programs may provide training prior to departure. Neurologists may specifically contribute by identifying patients with spinal cord and traumatic brain injuries, treating sentinel cases of meningitis, ${ }^{20}$ or assisting in early rehabilitation for patients with new-onset disability. In general, disaster relief requires extra skills and protection that other overseas experiences may not require. If disaster relief is of particular interest, establishing a skill set and enrolling in courses that train a multidisciplinary cadre of ready and able professionals is advantageous. Interactions with national embassies or consulates can provide detailed information on the level of safety to be expected in particular regions.

\section{What to know before you go}

International experiences require a sense of adventure as well as meticulous preparation (figure). In addition to negotiating time away, clinical coverage, and funding with one's home institution or practice, it is imperative to build strong ties with foreign colleagues before the trip. Arrangements for travel, housing, health insurance, and credentialing should be made well in advance. Some countries require a national medical license that must be issued prior to practicing. Liability risks and necessary malpractice coverage will vary for every situation, making it imperative to consult early with one's local and host institutions to make appropriate arrangements. It may be prudent to consult with a malpractice attorney well in advance of a trip to ensure adequate coverage. Depending on the location of the trip, antimalarial prophylaxis may be indicated, as might vaccinations or boosters for hepatitis, typhoid, yellow fever, and tetanus, among others. Some countries require proof of vaccination status before entry into the country. Immunization and antimalarial guidelines can be searched, by 
country, in the Center for Disease Control's Yellow Book (table 2). In case of emergency, a medical evacuation plan should be drafted and trips can be registered with the State Department. Passports and any necessary visas must be up-to-date, with available copies at home in case of loss or theft. Banks and credit card companies may require notification in advance of planned travel abroad. Some countries conduct daily affairs almost exclusively in cash.

Particularly when in-country time is limited, it is crucial to be familiar with the structure of the foreign health care system in advance, including the medical hierarchy and responsibilities, diagnostic and therapeutic capabilities, and contacts in the event of personal emergencies. Neurologists intending to bring supplies with them should check with the host institution first. Avoid bringing equipment that local providers lack the training to both use and repair independently.

\section{DISCUSSION}

LMICs are disproportionately affected by neurologic disorders compared to high-income populations, and underequipped to recognize and treat affected patients. Although we have focused exclusively on "low- and middle-income countries"- a term used by the World Bank based on gross national income per capita - it is possible that some countries do not need assistance in some well-served areas. It is also possible that high-income countries with rural, remote, and vulnerable populations also have many of the same needs as impoverished populations in LMICs. Interested neurologists can have a considerable effect through short- and longduration clinical service trips, visiting professorships and teaching conferences, international research collaborations, and disaster relief efforts. We must approach these partnerships with ample time, planning, understanding, and flexibility. We must be mindful of the setting, recognize our limitations, and engage patients and subjects with the highest ethical standards. Finally, we must not think of neurology as a luxury for developed countries. Public health and specialty care should be parallel, not serial, steps. ${ }^{21}$ Neurology is universal: the need is global.

\section{REFERENCES}

1. Murray CJ, Vos T, Lozano R, et al. Disability-adjusted life years (DALYs) for 291 diseases and injuries in 21 regions, 1990-2010: a systematic analysis for the global burden of disease study 2010. Lancet 2012;380:2197-2223.

2. Chin JH. Stroke in sub-Saharan Africa: an urgent call for prevention. Neurology 2012;78:1007-1008.

3. Usman U. International issues: neurology training in Pakistan: my experience as a neurology resident. Neurology 2009;72:e58-e60.

4. Birbeck GL, French JA, Perucca E, et al. Evidence-based guideline: antiepileptic drug selection for people with HIV/AIDS: report of the quality standards subcommittee of the American Academy of Neurology and the ad hoc task force of the commission on therapeutic strategies of the International League against Epilepsy. Neurology 2012;78:139-145.

5. Prevett M. Epilepsy in sub-Saharan Africa. Pract Neurol 2013;13:14-20.

6. Shibre T, Alem A, Tekle-Haimanot R, Medhin G, Tessema A, Jacobsson L. Community attitudes towards epilepsy in a rural Ethiopian setting: a re-visit after 15 years. Ethiop Med J 2008;46:251-259.

7. Jilek-Aall L, Rwiza HT. Prognosis of epilepsy in a rural African community: a 30-year follow-up of 164 patients in an outpatient clinic in rural Tanzania. Epilepsia 1992;33:645-650.

8. Rwiza HT, Matuja WB, Kilonzo GP, et al. Knowledge, attitude, and practice toward epilepsy among rural Tanzanian residents. Epilepsia 1993;34:1017-1023.

9. Bower JH, Zenebe G. Neurologic services in the nations of Africa. Neurology 2005;64:412-415.

10. Roberts M. A piece of my mind: duffle bag medicine. JAMA 2006;295:1491-1492.

11. Peluso MJ, Forrestel AK, Hafler JP, Rohrbaugh RM. Structured global health programs in U.S. medical schools: a web-based review of certificates, tracks, and concentrations. Acad Med 2013;88: 124-130.

12. Laccheo I, Espinosa PS. International issues: neurology mission in the Ecuadorian amazon rainforest. Neurology 2012;78:e60-e62.

13. Dahodwala N. Neurology education and global health: my rotation in Botswana. Neurology 2007;68: E15-E16.

14. Fleisher J. Reflections: neurology and the humanities: I knew that smile. Neurology 2013;80:e83.

15. Heatwole C. International education issues: practicing and teaching international neurology: the Krakow experience. Neurology 2007;69:1721-1723. 
16. Aarli JA, Abramsky O. When is a global health program global? Neurology 2013;80:2088-2089.

17. Medina MT, Munsat T. Neurology education in Latin America and the World Federation of Neurology. J Neurol Sci 2010;298:17-20.

18. Siddiqi OK, Koralnik IJ, Atadzhanov M, Birbeck GL. Emerging subspecialties in neurology: global health. Neurology 2013;80:e78-80.

19. American Medical Association. Opinion 2.077: ethical considerations in international research. Available at: http://www.ama-assn.org//ama/pub/physician-resources/medical-ethics/code-medical-ethics/ opinion2077.page. Accessed July 30, 2013.

20. Médecins sans frontières. Chad facing malnutrition and meningitis emergencies. Available at: http://www. doctorswithoutborders.org/press/release.cfm?id=5848\&cat=press-release. Accessed August 24, 2013.

21. Mateen FJ. International issues: neurology in Bangladesh. Neurology 2010;74:e5-e8.

\section{STUDY FUNDING}

No targeted funding reported.

\section{DISCLOSURES}

J. Fleisher has received an unrestricted educational grant from Medtronic and training grant T32-NS-061779 from the NIH. F. Mateen has received funding from the Canadian Institute of Health Research. Full disclosure form information provided by the authors is available with the full text of this article at Neurology.org/cp.

\section{Related articles from other AAN physician and patient resources}

\section{Neurology ${ }^{\circledR} \quad$ Neurology.org}

Neurology and international organizations July 23, 2013;81:392-394.

Creation of the AAN Global Health Section, Part I: Introduction and background May 28, 2013;80:2062-2064.

Creation of the AAN Global Health Section, Part II: Vision and goals June 4, 2013;80:2151-2153.

\section{Continuum $^{\circledR}$ • ContinuumJournal.com}

Neurocysticercosis

December 2012;1392-1416.

Infectious myelopathies

December 2012;1351-1373.

\section{Neurology Today ${ }^{\circledR} \quad$ - Neurotodayonline.com}

Neurologists Exit Syria as Hospital System Nears Collapse October 17, 2013;13:1-5.

Glaxo Under Fire Over MS Clinical Trial Allegations at R\&D Center in China September 5, 2013;13:42-43.

New Data to Support Combination Drug Therapy for Cryptococcal Meningitis May 16, 2013;13:6-7. 